\title{
Next generation pseudocapacitor materials from sol-gel derived transition metal oxides
}

\author{
Torsten Brezesinski · John Wang • \\ Sarah H. Tolbert • Bruce Dunn
}

Received: 2 January 2010/ Accepted: 12 February 2010/Published online: 24 February 2010

(C) The Author(s) 2010. This article is published with open access at Springerlink.com

\begin{abstract}
Capacitive energy storage is distinguished from other types of electrochemical energy storage by short charging times and the ability to deliver significantly more power than batteries. A key limitation to this technology is its low energy density and for this reason there is considerable interest in exploring pseudocapacitive charge storage mechanisms which offer the prospect of increasing energy density without compromising the power density of electrochemical capacitors. In this paper we review our recent work on using sol-gel synthesis methods to prepare nanostructured transition metal oxides which exhibit increased levels of pseudocapacitance and enhanced energy storage properties. Our work with $\mathrm{TiO}_{2}$ nanoparticles and mesoporous films of $\mathrm{TiO}_{2}$ and $\mathrm{CeO}_{2}$ is highlighted as we use these studies to understand the role of crystallite size, nanoscale porosity and understanding the differences between pseudocapacitance and intercalation processes.
\end{abstract}

T. Brezesinski

Institute of Physical Chemistry, Justus-Liebig-University

Giessen, Heinrich-Buff-Ring 58, 35392 Giessen, Germany

T. Brezesinski $\cdot$ S. H. Tolbert

Department of Chemistry and Biochemistry, University

of California at Los Angeles, Los Angeles, CA 90095, USA

B. Dunn

Department of Materials Science and Engineering, University

of California at Los Angeles, Los Angeles, CA 90095, USA

J. Wang

HRL Laboratories, LLC, Malibu, CA 90265, USA

S. H. Tolbert · B. Dunn $(\bowtie)$

California NanoSystems Institute, University of California

at Los Angeles, Los Angeles, CA 90095, USA

e-mail: bdunn@ucla.edu
Keywords $\mathrm{TiO}_{2}$ nanoparticles · Mesoporous materials . Capacitive energy storage - Electrochemical capacitors . Impedance spectroscopy

\section{Introduction}

In order for society to move towards a sustainable and renewable energy future, energy storage systems will be required to efficiently utilize the energy produced from intermittent sources such as from sun and wind. Among the different electrical energy storage technologies, batteries and electrochemical capacitors (ECs) are the most prominent with applications ranging from portable electronics to hybrid vehicles to large-scale power generation [1]. With the great interest in lithium-ion batteries over the past decade, ECs received much less attention until recently. There is now the recognition that ECs offer a number of desirable properties which can complement or replace batteries: fast charging (within seconds), reliability, longterm cycling ( $>500,000$ cycles), and the ability to deliver $>10$ times more power than batteries [2]. Moreover, it is apparent that there are unique opportunities for ECs, such as in energy recovery, where batteries are simply not appropriate. A good example is the use of capacitors to recover energy from repetitive processes (descending elevators, braking cars), applications enabled by their fast charging rate. The limiting feature that prevents more widespread usage of ECs has been the relatively low energy density of the materials used in capacitive storage applications.

ECs may be divided into two main categories based on their energy storage mechanism: electric double layer capacitors (EDLCs) and pseudocapacitors [3]. The former is based on storing electrical charge in a thin double layer 
at the interface between the electrode, typically carbon, and the electrolyte, while the latter involves reversible faradaic charge-transfer reactions. EDLCs have come to be known as supercapacitors or ultracapacitors because their energy density is orders of magnitude larger than conventional electrostatic or electrolytic capacitors [2]. The physical nature of the capacitive charge storage mechanism for EDLCs differs significantly from that of batteries (chemical energy storage) and leads to the advantageous operating characteristics mentioned above.

The interest in using pseudocapacitor based materials for electrochemical capacitors is that the energy density associated with faradaic reactions is much higher, by at least an order of magnitude, than traditional double layer capacitance (above $100 \mu \mathrm{F} / \mathrm{cm}^{2}$ for pseudocapacitance versus $10-20 \mu \mathrm{F} / \mathrm{cm}^{2}$ for EDLCs) [3]. Thus, by creating high surface area pseudocapacitor materials, it should be possible to greatly increase the specific capacitance to levels well above that of carbon.

In our research, we have focused on designing pseudocapacitor materials for improved energy storage. For this reason, we are investigating transition metal oxides in order to exploit their charge transfer properties. We are also emphasizing specific morphologies and perhaps the most obvious one is that of nanostructured materials. The reason is that when electrochemically active materials approach nanoscale dimensions, the charge storage from faradaic processes occurring at the surface of the material, referred to as the pseudocapacitive effect, becomes increasingly important $[4,5]$. Another interesting morphology is that of mesostructured materials where the use of a structuredirecting agent enables one to design the arrangement of inorganic-organic composites at the nanoscale. We are investigating mesoporous morphologies because of electrolyte access to the pore-solid architecture as well as enhanced oxidation and reduction kinetics due to the relatively short diffusion path length for ions and electrons [6]. In the present paper we review some of the highlights of our studies on nanoparticle $\mathrm{TiO}_{2}$ and mesoporous films of $\mathrm{TiO}_{2}$ and $\mathrm{CeO}_{2}[7,8]$. These materials serve as model systems in which to understand the distinction between pseudocapacitance and intercalation, the effect of crystallite size and the role of nanoscale porosity in improving charge storage kinetics.

\section{Pseudocapacitance of $\mathrm{TiO}_{2}$ nanoparticles and mesoporous films}

The starting point for our studies is the investigation of nanocrystalline particles of $\mathrm{TiO}_{2}$ (anatase). Although the material is known to be a reasonably attractive lithium ion insertion compound, we selected this material because researchers have begun to examine the fundamental question of how particle size and morphology influence its electrochemical properties $[9,10]$. In our studies, we used a detailed voltammetric analysis to establish quantitatively the dependence of the pseudocapacitance on the size of $\mathrm{TiO}_{2}$ crystallites [7]. Phase-pure nanocrystalline $\mathrm{TiO}_{2}$ (anatase) was prepared by modifying the synthesis method reported by Niederberger et al. [11]. Monodisperse nanoparticles $(\leq 10 \mathrm{~nm})$ were prepared without surfactants and with all organic constituents removed. For the electrochemical studies, we developed methods using glassy carbon electrodes which did not require conductive agents or polymeric binders, so that the experimental results more accurately represented the fundamental electrochemical properties of only the nanodimensional $\mathrm{TiO}_{2}$ material.

In our analysis, cyclic voltammetry (CV) was used to characterize charge storage behavior (Fig. 1). The area underneath the curves corresponds to the total stored charge, which is related to the concentration of $\mathrm{Li}^{+}$-ions in the anatase $\mathrm{TiO}_{2}$ film. The cathodic/anodic peaks represent the lithium insertion/extraction behavior in the anatase lattice and occur at 1.7 and $2.0 \mathrm{~V}$ versus $\mathrm{Li} / \mathrm{Li}^{+}$, respectively. This lithium insertion process is often expressed by the equation

$\mathrm{TiO}_{2}+x \mathrm{Li}^{+}+x \mathrm{e}^{-} \leftrightarrow \mathrm{Li}_{x} \mathrm{TiO}_{2}$

The mole fraction of inserted lithium, $x$, is generally close to 0.5 for the anatase structure. This value of $x$ corresponds to a specific capacity of $168 \mathrm{mAh} / \mathrm{g}$ [12-14].

The total stored charge can be separated into three components: the faradaic contribution from the $\mathrm{Li}^{+}$ion insertion process, the faradaic contribution from the charge transfer process with surface atoms, referred to as pseudocapacitance, and the non-faradaic contribution from the double layer effect [15]. In our studies we developed a methodology which enabled us to distinguish between the currents arising from lithium ion insertion and those occurring from capacitive processes.

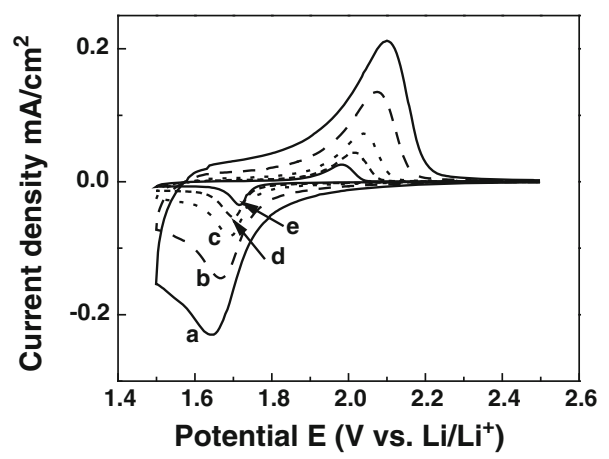

Fig. 1 Voltammetric response for $7 \mathrm{~nm} \quad \mathrm{TiO}_{2}$ nanocrystallites. Reprinted with permission [7] 
Our approach is based on characterizing the sweep rate dependence on the current. For systems which exhibit diffusion-controlled behavior, the current $(i)$ scales with $v^{1 / 2}$ in the following equation [16]:

$i=n F A C * D^{1 / 2} v^{1 / 2}(\alpha n F / R T)^{1 / 2} \pi^{1 / 2} \chi(\mathrm{bt})$

where $C^{*}$ is the surface concentration of the electrode material, $\alpha$ is the transfer coefficient, $D$ is the chemical diffusion coefficient, $n$ is the number of electrons involved in the electrode reaction, $A$ is the surface area of the electrode materials, $F$ is the Faraday constant, $R$ is the molar gas constant, $T$ is the temperature, and the function $\chi(\mathrm{bt})$ represents the normalized current for a totally irreversible system as indicated by the $\mathrm{CV}$ response [16]. This $v^{1 / 2}$ response is indicative of faradaic intercalation reactions. By comparison, capacitive systems are characterized by having the current proportional to the sweep rate:

$i=v C_{d} A$

where $C_{d}$ is the capacitance [14].

Thus, we can express the total current in terms of two separate processes,

$i(V)=k_{1} v+k_{2} v^{1 / 2}$

where, $k_{1} v$ and $k_{2} v^{1 / 2}$ correspond to the current contributions from surface capacitive effects and diffusion controlled intercalation processes, respectively. Thus, by determining $k_{1}$ and $k_{2}$, we are able to quantify, at specific potentials, the fraction of the current due to each of the processes. The results are summarized in Table 1 where we compare the influence of $\mathrm{TiO}_{2}$ crystallite size on charge storage properties [7]. Both the total stored charge and the capacitive contribution to the stored charge increase with decreasing particle size. Because of this, smaller crystallites exhibit faster charge/discharge kinetics. The capacitance contribution includes both double layer and pseudocapacitive effects. However, because the area normalized capacitance is well above $100 \mu \mathrm{F} / \mathrm{cm}^{2}$, it is evident that this contribution is pseudocapacitive in nature. It is interesting that the amount of charge stored from lithium intercalation processes decreases as particles become smaller, however, the increased capacitive contribution for

Table 1 Dependence of physical properties and charge storage properties on crystallite size

\begin{tabular}{lrrr}
\hline & $7 \mathrm{~nm}$ & $10 \mathrm{~nm}$ & $30 \mathrm{~nm}$ \\
\hline Total stored charge (at $0.5 \mathrm{mV} / \mathrm{s} ; \mathrm{C} / \mathrm{g})$ & 490 & 460 & 420 \\
Percent capacitive contribution $(\%)$ & 55 & 35 & 15 \\
Surface normalized capacitance $\left(\mu \mathrm{F} / \mathrm{cm}^{2}\right)$ & 120 & 110 & 130 \\
Lithium intercalation capacity $(\mathrm{mAh} / \mathrm{g})$ & 61 & 83 & 100 \\
\hline
\end{tabular}

Reprinted with permission [7] the small particles more than compensates for this decrease. These results suggest that charge stored at the surface of the $\mathrm{TiO}_{2}$ from the pseudocapacitance storage mechanism can be an important factor in achieving high values for the gravimetric energy density.

In a related study, we investigated the development of pseudocapacitive charge storage in mesoporous films of $\mathrm{TiO}_{2}$ [17]. One of the principal goals of this work was to determine whether mesoporous films could exhibit the high level of charge storage and fast kinetics of the $\mathrm{TiO}_{2}$ nanoparticle films described above. As indicated previously, a clear benefit of the mesoporous morphology is that the interconnected mesoscale porosity allows facile electrolyte diffusion throughout the material, enabling the electrolyte to access the redox-active pore walls. As for the solid phase, the crystallite sizes of the pore walls are typically in the same nanodimensional size range as that of the nanoparticles.

The polymer templating of inorganic materials has been widely used to prepare mesoporous materials with ordered pore-solid architectures both as powders and thin films [18, 19]. A limitation with this technique, the inability to retain nanoscale order upon crystallization of the inorganic walls, has recently been overcome through the use of appropriate diblock copolymers [20]. In the research reported here, we used a large poly(ethylene-co-butylene)- $b$-poly(ethylene oxide) diblock copolymer (also referred to as KLE), which allows the formation of cubic architectures with 20-30 nm pore-to-pore distances and 10-15 nm thick walls [21]. These thicker walls permit stable crystallites to be formed without distorting the pore network. We have applied this synthesis approach to prepare mesoporous transition metal oxide films in which either sol-gel reagents or preformed nanocrystals were used as building blocks to form the inorganic walls [7, 8]. In some instances the mesoporous materials exhibited iso-oriented nanocrystalline domains [22].

The mesoporous thin films were prepared by coassembly of inorganic oligomers with KLE diblock copolymer as the structure-directing agent using an evaporation induced self-assembly (EISA) process [23]. The mesoporous films that used $\mathrm{TiO}_{2}$ nanoparticles as building blocks produced pore walls with nanoscale porosity while films that used $\mathrm{TiCl}_{4}$ as the precursor lead to materials with dense walls. The overall charge storage kinetics shown in Fig. 2 indicate that mesoporous $\mathrm{TiO}_{2}$ films can indeed exhibit the same kinetics and amount of charge storage as that of $\mathrm{TiO}_{2}$ nanoparticles. Interestingly, the mesoporous film prepared from $\mathrm{TiO}_{2}$ nanoparticles has much better charge storage properties and faster kinetics than the films prepared from $\mathrm{TiCl}_{4}$. When we use our analysis to separate the charge storage into diffusion-controlled and capacitive contributions (Table 2), we find the reason for this 


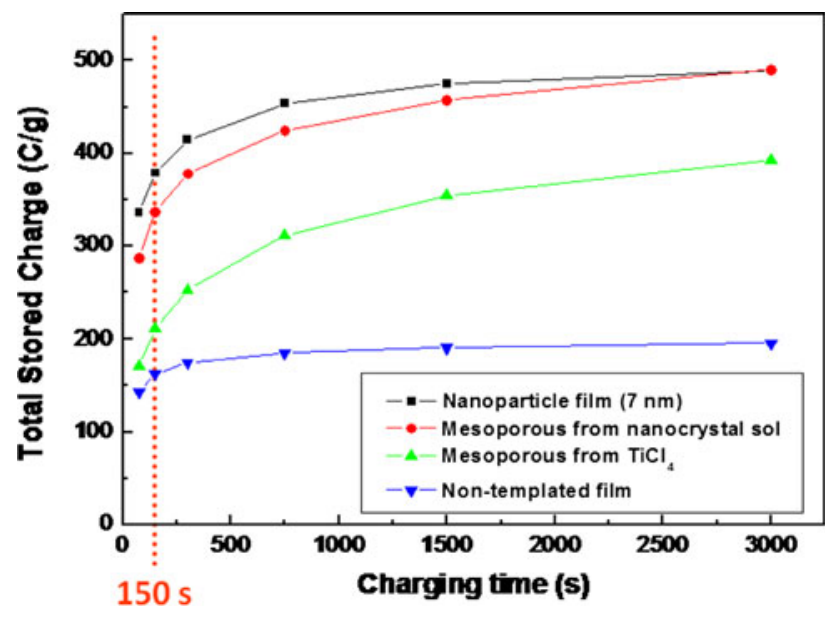

Fig. 2 A comparison of charging rates for $\mathrm{TiO}_{2}$ nanoparticle films, two different mesoporous films and non-templated films

difference in charge storage. That is, the mesoporous films prepared from $\mathrm{TiCl}_{4}$ have less capacitive storage compared to the mesoporous films prepared using the $\mathrm{TiO}_{2}$ nanoparticles. Finally, we note that when nanocrystal based films are formed without mesoscale porosity, a high fraction of the total stored charge is capacitive, however, the total capacity is low. One reason for this is that most of the film is not accessible to the electrolyte/solvent.

These results show the nature of pseudocapacitive behavior that develops in high surface area mesoporous oxide films. Our data suggest that a combination of both the mesoporous morphology and the use of nanocrystals as the basic building blocks offers a very promising approach for creating metal oxide pseudocapacitors that can be utilized as electrode materials for electrochemical capacitive storage [17].

\section{Electrochemical impedance spectroscopy of pseudocapacitive materials}

We have carried out electrochemical impedance spectroscopy (EIS) to gain additional insight into the pseudocapacitive contributions to the electrochemical capacitance. Our first studies were with $\mathrm{TiO}_{2}$ nanoparticle films where our objective was to characterize the faradaic contributions to the charge-transfer process and to determine the influence of crystallite size. The impedance spectrum for $\mathrm{TiO}_{2}$ films comprised of $7 \mathrm{~nm}$ crystallites is shown in Fig. 3a while the equivalent circuit model used to analyze the spectrum is displayed in Fig. $3 \mathrm{~b}$.

In the frequency range below $10 \mathrm{~Hz}$, the impedance is dominated by the constant phase element, $\mathrm{CPE}_{\text {echem }}$, in parallel with a resistance, $R_{\text {leak }}$. The constant phase element is used to model behavior that deviates from an ideal capacitor. The impedance, $Z_{\mathrm{CPE}}$, is expressed as

$Z_{\mathrm{CPE}}=\left[B(j \omega)^{n}\right]^{-1}$

where $B$ and $n(0<n<1)$ are frequency independent proportionality constants [24]. When $n=1$, it is an ideal capacitor and the CPE can be expressed as a capacitance. The $\mathrm{CPE}_{\text {echem }}$ represents the electrochemical capacitance of a porous electrode, and the $R_{\text {leak }}$ in parallel signifies a small leakage current at the electrode/electrolyte interface.

Table 2 Comparison of the electrochemical storage properties for $\mathrm{TiO}_{2}$ nanoparticle films, mesoporous films and non-templated films

\begin{tabular}{lrrrr}
\hline & $7 \mathrm{~nm}$ & Meso-NP & Meso-TiCl $_{4}$ & Non-temp \\
\hline Total stored charge (at $0.5 \mathrm{mV} / \mathrm{s} ; \mathrm{C} / \mathrm{g})$ & 490 & 490 & 390 & 200 \\
Percent capacitive contribution (\%) & 55 & 45 & 35 & 50 \\
Lithium intercalation capacity (C/g) & 220 & 270 & 250 & 100 \\
\hline
\end{tabular}

Fig. 3 Electrochemical impedance spectroscopy for $\mathrm{TiO}_{2}$ nanoparticle films using $7 \mathrm{~nm}$ crystallites. a Nyquist representation $(0.1 \mathrm{~Hz}$ to $100 \mathrm{kHz}$ ) at 1.5 and $1.75 \mathrm{~V}$ versus $\mathrm{Li}^{+} / \mathrm{Li}$. b Equivalent circuit model for $\mathrm{TiO}_{2}$ nanoparticle films. Reprinted with permission [7]

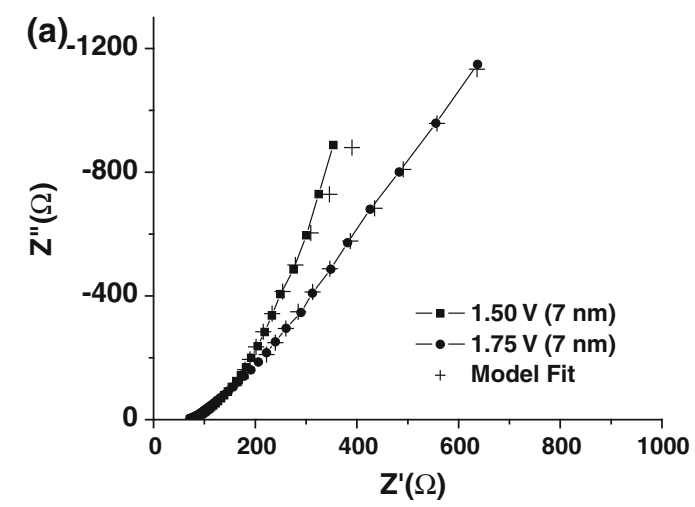

(b)

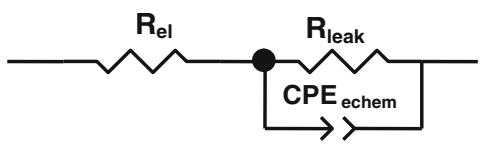




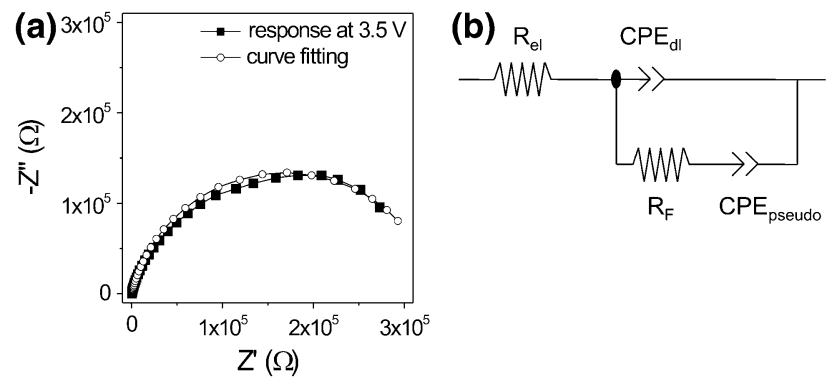

Fig. 4 Electrochemical impedance spectroscopy for mesoporous $\mathrm{CeO}_{2}$ films. a Nyquist representation at $3.5 \mathrm{~V}$ when $\mathrm{Ce}$ is fully oxidized. b Equivalent circuit model for mesoporous $\mathrm{CeO}_{2}$ films. Reprinted with permission [8]

We used numerical fitting of the equivalent circuit model to determine the value for each circuit element for the film [7]. Although we were able to determine the specific capacitance for the film, we were not able to unambiguously separate faradaic (pseudcapacitance) and non-faradaic (double layer) contributions. We were, however, able to compare specific capacitance values for films prepared using different crystallite sizes. The results from EIS were consistent with the voltammetry analysis in that higher capacitance was observed with films composed of the smaller $\mathrm{TiO}_{2}$ nanocrystals.

We have also used EIS to characterize the capacitive behavior of mesoporous $\mathrm{CeO}_{2}$ films. The impedance spectra are very different depending upon the potential (i.e., whether $\mathrm{Ce}^{4+}$ is reduced to $\mathrm{Ce}^{3+}$ ). The complex impedance spectrum at $3.5 \mathrm{~V}$, when nearly all the cerium is in the oxidized state, is shown in Fig. 4a. Upon reduction, the spectrum becomes similar to that of the $\mathrm{TiO}_{2}$ nanoparticle film (Fig. 3a).

The circuit model shown in Fig. $4 \mathrm{~b}$ is able to provide considerable insight concerning the different capacitive contributions to the mesoprous $\mathrm{CeO}_{2}$ film. $R_{\mathrm{el}}$ and $R_{\mathrm{F}}$ represent the resistance of the electrolyte solution and the faradaic charge transfer resistance, respectively [25]. Because pseudocapacitance arises from a potential-dependent interfacial redox reaction, $R_{\mathrm{F}}$ is in series with a

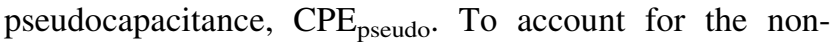
faradaic current for double-layer charging, $\mathrm{CPE}_{\mathrm{dl}}$ is in parallel with the faradaic charge transfer resistance.

Numerical fitting to the circuit model enables us to separate double-layer and pseudocapacitive contributions as a function of potential (Fig. 5). At potentials above $3 \mathrm{~V}$, there is no pseudocapacitive contribution because nearly all the $\mathrm{Ce}$ is in the fully oxidized state. There is only a small double-layer capacitance. At lower potentials, $\mathrm{Ce}^{4+}$ reduces to $\mathrm{Ce}^{3+}$ and pseudocapacitance develops as the increasing electronic conductivity enables charge transfer reactions to occur. At these potentials, pseudocapacitive contributions increase with decreasing voltage while

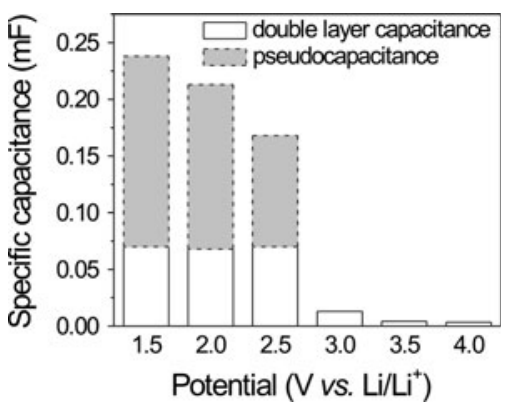

Fig. 5 Dependence of specific capacitance on double-layer and pseudocapacitance processes as a function of potential as determined by numerical fitting to the equivalent circuit. Reprinted with permission [8]

double layer contributions are mostly voltage independent. The value of $n$ for $\mathrm{CPE}_{\text {pseudo }}$ is greater than 0.9 , so that if a simple capacitor is substituted for $Z_{\mathrm{CPE}}$ in the circuit, the results are qualitatively similar with capacitance values changing by only $\sim 10 \%$.

These results demonstrate the feasibility of using EIS to effectively separate double layer and pseudocapacitive contributions so that we can better understand the means by which mesoporous materials develop high levels of charge storage.

\section{Conclusions}

Although pseudocapacitive processes are attractive for capacitive storage, these materials have yet to achieve the specific capacitance levels expected for 1-electron or 2 -electron redox reactions. The present paper suggests that there are certain 'design rules' for pseudocapacitor materials. Our analytical methods are very helpful here because we are able to distinguish between capacitive processes and slower diffusion-controlled processes. The combination of nanocrystals and mesoporosity is beneficial as it leads to considerable enhancement in capacitive energy storage. In general, the use of hierarchical architectures where one forms nanostructured building blocks and then assembles these blocks into a mesoporous structure may emerge as a generalized approach which enables inorganic materials to attain greater energy density without compromising power density.

Acknowledgments This research was supported by the Office of Naval Research (B.D. and S.H.T), the National Science Foundation under grant CHE-0527015 (S.H.T.) and the Fonds der Chemischen Industrie (Liebig Fellowship, T.B.)

Open Access This article is distributed under the terms of the Creative Commons Attribution Noncommercial License which permits any noncommercial use, distribution, and reproduction in any medium, provided the original author(s) and source are credited. 


\section{References}

1. Simon P, Gogotsi Y (2008) Nature Mater 7:845-850

2. Miller JR, Simon P (2008) Science 321:651-652

3. Conway BE, Pell WG (2003) J Solid State Electrochem 7:637644

4. Jamnik J, Maier J (2003) Phys Chem Chem Phys 5:5215-5220

5. Balaya P, Bhattacharyya AJ, Jamnik J, Zhukovskii YF, Kotomin EA, Maier J (2006) J Power Sources 159:171-178

6. Cheng W, Baudrin E, Dunn B, Zink JI (2001) J Mater Chem 11:92-97

7. Wang J, Polleux J, Lim J, Dunn B (2007) J Phys Chem C 111:14925-14931

8. Brezesinski T, Wang J, Brezesinski K, Senter R, Dunn B, Tolbert SH (2010) ACS NANO. doi:10.1021/nn9007324

9. Kavan L, Rathousky J, Gratzel M, Shklover V, Zukal A (2000) J Phys Chem B 104:12012-12020

10. Sudan G, Baudrin E, Larcher D, Tarascon JM (2001) J Mater Chem 15:1263-1270

11. Niederberger M, Bartl MH, Stucky GD (2002) Chem Mater 14:4364-4370

12. Jiang CH, Wei MD, Qi ZM, Kudo T, Honma I, Zhou HS (2007) J Power Sources 166:239-243

13. Kavan L, Kalbac M, Zukalova M, Exnar I, Lorenzen V, Nesper R, Graetzel M (2004) Chem Mater 16:477-485
14. Lindstrom H, Sodergren S, Solbrand A, Rensmo H, Hjelm J, Hagfeldt A, Lindquist SE (1997) J Phys Chem B 101:7717-7722

15. Conway BE, Birss V, Wojtowicz J (1997) J Power Sources 66:1-14

16. Bard AJ, Faulkner LR (1980) Electrochemical methods: fundamentals and applications. John Wiley, New York

17. Brezesinski T, Wang J, Polleux J, Dunn B, Tolbert SH (2009) J Am Chem Soc 131:1802-1809

18. Brezesinski T, Groenewolt M, Antonietti M, Smarsly B (2006) Angew Chem Int Ed 4:781-785

19. Sun D, Riley AE, Cadby AJ, Richman EK, Korlann SD, Tolbert SH (2006) Nature 441:1126-1130

20. Thomas A, Schlaad H, Smarsly B, Antonietti M (2003) Langmuir 19:4455-4459

21. Sanchez C, Boissiere C, Grosso D, Laberty C, Nicole L (2008) Chem Mater 20:682-737

22. Brezesinski T, Wang J, Tolbert SH, Dunn B (2010) Nat Mater (in press)

23. Brinker CJ, Lu YF, Sellinger A, Fan HY (1999) Adv. Mater 11:579-585

24. Brett CMA, Brett AMO (1993) Electrochemistry: principles, methods, and applications. Oxford University Press, New York

25. Conway BE (1997) Electrochemical supercapacitors: scientific fundamentals and technological applications. Kluwar, New York 